\title{
RAMSEY GAMES
}

BY

\author{
A. HAJNAL AND ZS. NAGY
}

\begin{abstract}
The paper deals with game-theoretic versions of the partition relations $\alpha \rightarrow(\beta)_{2}^{<\tau}$ and $\alpha \rightarrow(\beta)_{2}^{\tau}$ introduced in [2]. The main results are summarized in the Introduction.
\end{abstract}

0. Introduction. In their paper [2], Baumgartner, Galvin, McKenzie and Laver introduced a new game. Let $\alpha, \beta$ be ordinals, $\tau$ a cardinal. The Ramsey game $R(\alpha, \tau, \beta)$ is defined as follows. There are two players, White and Black, who alternately pick previously unchosen members of $[\alpha]^{\tau}$. At limit stages it is of course White's turn to move. The game ends when the set $[\alpha]^{\tau}$ is exhausted. White wins if there is a set $A \subset \alpha, \operatorname{tp} A=\beta$ with $[A]^{\tau} \subset W$, where $W \subset[\alpha]^{\tau}$ is the set of $\tau$ element sets which White chose; otherwise Black wins. We say that White (Black) wins $R(\alpha, \tau, \beta)$ if White (Black) has a winning strategy.

This game can be considered as a game-theoretic version of the partition relation $\alpha \rightarrow(\beta)_{2}^{\tau}$. The game $R(\alpha,<\tau, \beta)$ is defined similarly, where the choices are made from $[\alpha]^{<\tau}$ and to win White must get an $A \subset \alpha$ with $[A]^{<\tau} \subset W$ and $\operatorname{tp} A=\beta$.

It is also clear that more general games can be defined, where $\alpha, \beta$ are order types or even more generally $\beta$ can be the isomorphism type of certain objects, say that of complete bipartite graphs. Our main results say something about the games $R(\alpha, \tau, \beta)$ and $R(\alpha,<\tau, \beta)$ as defined above, but we will use the more general games in establishing some lemmas. We hope that the symbols used there have a self-explanatory meaning.

We remark that in [2] the games were defined to last $|\alpha|$ moves. Our present definition seems to be technically more convenient, and the results of [2] remain valid.

In $[2]$ it was proved that White wins $R(\omega,<r+1, \omega)$ for $r<\omega$. However the proof given there yields the following

THEOREM [2]. Assume $\kappa \geq \omega, 0<r<\omega$ and $\kappa \rightarrow(\beta)_{2^{r-1}}^{r}$. Then White wins $R(\kappa, \leq r, \beta)$.

This shows that for the games $R(\kappa, \tau, \beta)$ we can hope for interesting new results only if $\kappa \nrightarrow(\beta)_{2}^{\tau}$ holds.

Our main results are the following:

(1) Assume $2^{\kappa}=\kappa^{+}$. Then Black wins $R\left(\kappa^{+}, 2, \kappa^{+}\right)$(see Theorem 1). Our proof shows that it is consistent with ZFC that $2^{\kappa}>\kappa^{+}$and Black still wins $R\left(\kappa^{+}, 2, \kappa^{+}\right)$ but we have no other information.

Problem 1. Is it a theorem of ZFC that Black wins $R\left(2^{\aleph_{0}}, 2, \aleph_{1}\right)$ ?

Received by the editors April 26, 1982 and, in revised form, August 18, 1983.

1980 Mathematics Subject Classification. Primary 04A20; Secondary 03E55.

(C) 1984 American Mathematical Society $0002-9947 / 84 \$ 1.00+\$ .25$ per page 
For $2<\tau=r<\omega$ we have even less information and we only state the simplest case of our results. Using a result of $\mathrm{R}$. Laver we can prove

(2) Assume $2^{\aleph_{0}}=\aleph_{1}$ and the negation of Chang's conjecture holds, i.e. $\aleph_{2} \nrightarrow$ $\left[\aleph_{1}\right]_{\aleph_{1}, \aleph_{0}}^{2}$ is true. Then Black wins $R\left(\aleph_{2}, 3, \aleph_{1}\right)$ (see Theorem 2).

Problem 2. Does $2^{\aleph_{0}}=\aleph_{1} \wedge 2^{\aleph_{1}}=\aleph_{2}$ imply that Black wins $R\left(\aleph_{2}, 3, \aleph_{1}\right)$ ?

Problem 3. Is it consistent (with G.C.H.) that Black wins $R\left(\aleph_{3}, 4, \aleph_{1}\right)$ ?

ADDED IN PROOF. The first author and P. Komjath recently proved that it is consistent with ZFC + G.C.H. that Black wins $R\left(\aleph_{n}, n+1, \aleph_{1}\right)$ for $n<\omega$.

This should be compared with the known partition relation

$$
2^{2^{2^{\aleph_{0}}}} \nrightarrow\left(\aleph_{1}\right)_{2}^{4}
$$

In $\S 1$ we formulate a general set-theoretic principle which implies that Black wins $R\left(\aleph_{r}, r+1, \aleph_{1}\right)$ for $r<\omega$ as expected. We conjecture that this principle holds in $L$ or is at least consistent with ZFC + G.C.H. but we cannot prove it.

In case $\kappa$ is a limit cardinal, we have a result which shows another phenomenon.

(3) Assume $\kappa$ is a singular strong limit cardinal. Then White wins $R(\kappa, 2, \kappa)$ (see Theorem 3).

Note that say $\aleph_{\omega} \rightarrow\left(\aleph_{\omega}\right)_{2}^{2}$ is trivially false. On the other hand we cannot generalize (3) for larger $\tau$.

Problem 4. Assume $\aleph_{\omega}$ is a strong limit cardinal. Does White win the game $R\left(\aleph_{\omega}, 3, \aleph_{\omega}\right)$ ?

Note that, by an old result of Erdös and Rado [5], $\kappa \nrightarrow(\tau)_{2}^{\tau}$ holds for all $\tau \geq \omega$ and for all $\kappa$. This result has an easy generalization.

(4) For $\kappa \geq \tau \geq \omega$ Black wins the game $R(\kappa, \tau, \tau)$.

The proof of this will be left to the reader.

To explain our results concerning the games $R(\kappa,<\omega, \beta)$ we recall a definition: Free $_{\lambda}(\kappa,<\omega, \beta)$ means that the following statement is true: Whenever $F:[\kappa]^{<\omega} \rightarrow$ $[\kappa] \leq \lambda$, i.e. $F$ is a set mapping of finite type and order at most $\lambda$, then there is a set $H \subset \kappa \wedge \operatorname{tp} H=\beta$ such that $H$ is free for $F$. This means that, for each $X \in[H]^{<\omega}, F(X) \cap H \subset X$.

Results in $\left[\mathbf{1}, 3\right.$ and 4] say that if $V=L$, then for $\beta<\omega_{1} \beta$ limit and, for $1 \leq \lambda \leq \omega$, Free ${ }_{\lambda}(\kappa<\omega, \beta)$ holds iff $\kappa \rightarrow(\beta)_{2}^{<\omega}$ holds, moreover Free $\kappa_{0}\left(\kappa,<\omega, \omega_{1}\right)$ implies that $0 \#$ exists.

We will prove

(5) If Free ${ }_{1}(\kappa,<\omega, \beta)$ is false for a cardinal $\kappa$ and a limit ordinal $\beta$, then Black wins $R(\kappa,<\omega, \beta)$ (see Theorem 4$)$.

This proof uses remarks made by F. Galvin and A. Máté and is included here with their permission.

On the other hand we prove

(6) If $\kappa \rightarrow(\beta)_{2}^{<\omega}$ holds for a limit ordinal $\beta$, then White wins the game $R(\kappa,<\omega, \beta)$ (see Theorem 6 ).

This answers a problem asked in [2]. Note that this is a generalization of the result of $[2]$ as stated earlier but its proof is by no means simple.

We first proved that White wins $R(\kappa,<\omega, \kappa)$ for a measurable cardinal $\kappa$. Later P. Komjath [7] found a simple strategy for White in this game. We could use this strategy (with some modifications and tricks) to establish our result (6) for $\beta>\omega$. 
However for $\beta=\omega$, which is probably the most interesting case, we need a different argument. In $\S 2$ we define the concept of a sequoia, which we hope is of some independent interest and we establish special properties of these objects. The proof of (6) then falls into two parts.

We will first show that White has a strategy in the game $R(\kappa,<\omega, \beta)$ to pick all elements of a suitable sequoia, and after that we show that under the assumption $\kappa \rightarrow(\beta)_{2}^{<\omega}$ this sequoia contains all finite subsets of a set $B$ of type $\beta$.

1. The games $R(\kappa, r, \beta)$ for $2 \leq r<\omega$.

DEFINITION. Let $\kappa, \lambda, \tau$ be cardinals.

(i) We will call a mapping $F:[\kappa]^{<\omega} \rightarrow\left[[\kappa]^{\tau}\right]^{\leq \lambda}$ a $\tau$-set mapping of type $<\omega$ and of order $\leq \lambda$ on $\kappa$ if $A \cap X=\emptyset$ for all $A \in F(X), X \in[\kappa]^{<\omega}$. $\tau$-set mappings of type $n$ are defined similarly. In this case $D(F)$, the domain of $F$, is $[\kappa]^{n}$.

(ii) A subset $H \subset \kappa$ is said to be free for a $\tau$-set mapping $F$ on $\kappa$ iff for all $X \subset H$, such that $X \in D(F)$ and for all $A \in F(X), A \not \subset H$ holds.

(iii) Free ${ }_{\lambda}^{*}(\kappa,<\omega, \beta, \tau)$ (Free $\left.{ }_{\lambda}^{*}(\kappa, n, \beta, \tau)\right)$ denotes the following statement.

For all $\tau$-set mappings $F$ of type $<\omega$ (of type $n$ ) and of order $\leq \lambda$ there is a free set $H \subset \kappa$ with $\operatorname{tp} H=\beta$.

It is clear from the definition that 1-set mappings can be canonically identified to set mappings and that Free ${ }_{\lambda}^{*}(\kappa, \cdot, \beta, \tau)$ implies Free $_{\lambda}^{*}\left(\kappa, \cdot, \beta, \tau^{\prime}\right)$ for $1 \leq \tau<\tau^{\prime}$.

In this paper we do not intend to study this relation in general. The idea is that the statement $\neg \operatorname{Free}_{\lambda}^{*}(\kappa, \cdot, \beta, \lambda)$ in many cases yields winning strategies for Black.

Before showing that we define another statement.

DEFINITION. Let $\lambda \geq \omega . L(\kappa,<\omega, \lambda)(L(\kappa, n, \lambda))$ is the following statement. There is a $\lambda$ set mapping $F$ of type $<\omega$ (of type $n$ ) of order $\leq \lambda$ on $\kappa$ such that for all $D \subset \kappa$ with tp $D=\lambda^{+}\left(\forall A \in[D]^{\lambda} \exists X(X \in D(F) \wedge X \subset D \wedge A \in F(X))\right.$ holds.

It is again clear from the definition that $L(\kappa, \cdot, \lambda) \Rightarrow \neg$Free $_{\lambda}^{*}\left(\kappa, \cdot, \lambda^{+}, \lambda\right)$, moreover it is also clear that $L(\kappa, \cdot, \lambda)$ implies $2^{\lambda}=\lambda^{+}$provided $\kappa \geq \lambda^{+}$.

LEMMA 0. $2^{\lambda}=\lambda^{+}$implies $L\left(\lambda^{+}, 1, \lambda\right)$.

Proof. Let $\left[\lambda^{+}\right]^{\lambda}=\left\{A_{\alpha}: \alpha<\lambda^{+}\right\}$be a well-ordering of type $\lambda^{+}$of $\left[\lambda^{+}\right]^{\lambda}$. For $\alpha<\lambda^{+}$let $F(\alpha)=\left\{A_{\beta}: \beta<\alpha \wedge \alpha \notin A_{\beta}\right\}$.

LEMMA 1. Assume $\kappa>\lambda \geq \omega>r \geq 1$ and $\neg \operatorname{Free}_{\lambda}^{*}\left(\kappa, r, \lambda^{+}, \lambda\right)$. Then Black wins $R\left(\kappa, r+1, \lambda^{+}\right)$.

Proof. For $X \in[\kappa]^{r+1}$ let $X=\left\{x_{0}, \ldots, x_{r}\right\}, x_{0}<\cdots<x_{r} ;$ and $X^{(i)}=X \backslash\left\{x_{i}\right\}$ for $i \leq r$. Let $F:[\kappa]^{r} \rightarrow\left[[\kappa]^{\lambda}\right] \leq \lambda$ be a $\lambda$-set mapping establishing the assumption.

For each $Y \in[\kappa]^{r}$ let $F(Y)=\left\{F_{\nu}(Y): \nu<\lambda\right\}$ be an enumeration of $F(Y)$ possibly with repetitions.

Finally let $\varphi: \kappa \rightarrow[\kappa]^{r+1}$ be a coding function satisfying the following requirements:

(7) (i) $\varphi(\alpha) \cap \varphi(\beta)=\emptyset$ for $\alpha \neq \beta<\kappa$,

(ii) $\bigcup\{\varphi(\alpha): \alpha<\kappa\}=\kappa$,

(iii) $\alpha \subset \bigcap \varphi(\alpha) \wedge \varphi(\alpha) \subset \alpha+\omega$ for $\alpha<\kappa$.

Black's strategy will be the following. Assume $\gamma<\kappa$. When Black makes his (hers) $\gamma$ th move, he (she) looks at White's $\alpha$ th move, $W(\alpha)$, where $\alpha$ is the unique ordinal for which $\gamma$ is the $i$ th element of $\varphi(\alpha)$ for some $i \leq r$. 
He now considers the smallest $\nu<\lambda$ for which he did not choose earlier a set of form $\{x\} \cup W(\alpha)^{(i)}, x \in F_{\nu}\left(W(\alpha)^{(i)}\right)$. If such a $\nu$ exists, he chooses a set of the above form, otherwise he chooses the first element of $[\kappa]^{r+1}$ at his disposal in a well-ordering of type $\kappa$ of $[\kappa]^{r+1}$.

Assume now that a game was played and Black played according to his strategy. Let $D \in[\kappa]^{\lambda^{+}}$. By the assumption on $F$, there are $Y \in[D]^{r}$ and $\nu<\lambda$ such that

$$
F_{\nu}(Y) \subset D \text { and } F_{\nu}(Y) \cap Y=\emptyset .
$$

In case White chose less than $\lambda, r+1$ element sets containing $Y$ during the game, we clearly have $D \not \subset W$. Assume $\left\{\alpha_{\nu}: \nu<\lambda\right\}$ are the first $\lambda$ ordinals in increasing order for which White has picked an $r+1$ element subset containing $Y$. For some $i_{\nu}, Y=W\left(\alpha_{\nu}\right)^{\left(i_{\nu}\right)}$.

By (7), $\varphi\left(\alpha_{\nu}\right)^{\left(i_{\nu}\right)}<\alpha_{\nu}+\omega$. Until this move White has chosen less than $\lambda$ sets of the form $\{x\} \cup Y$ with $x \in F_{\nu}(Y)$ so when playing this move Black either chooses a set $\{x\} \cup Y$ with $x \in F_{\nu}(Y)$ or he must have done so in some earlier move.

LEMMA 2 (LAVER). Assume $\lambda \geq \omega, 2^{\lambda}=\lambda^{+}$and $\lambda^{++} \nrightarrow\left[\lambda^{+}\right]_{\lambda^{+}, \lambda}^{2}$, i.e. the $\lambda$-Chang conjecture fails. Then $L\left(\lambda^{++}, 2, \lambda\right)$ is true.

ProOF. Let $f:\left[\lambda^{++}\right]^{2} \rightarrow \lambda^{+}$establish the assumption, i.e.

$$
\forall D \in\left[\lambda^{++}\right]^{\lambda^{+}} \forall \mu<\lambda^{+} \exists X \in[D]^{2} f(X) \geq \mu .
$$

It is obvious that $f$ can be chosen so that it satisfies

$$
\forall \beta, \gamma<\alpha f(\{\alpha, \beta\}) \neq f(\{\alpha, \gamma\}) \text { for } \alpha<\lambda^{++} .
$$

By the assumption $2^{\lambda}=\lambda^{+}$, for each $\lambda \leq \alpha<\lambda^{++}$we can write

$$
[\alpha]^{\lambda}=\left\{A_{\nu}^{\alpha}: \nu<\lambda^{+}\right\} .
$$

Let $\beta<\alpha<\lambda^{++}$. For $\alpha<\lambda$ let $F(\{\alpha, \beta\})=\emptyset$ and for $\alpha \geq \lambda$ let

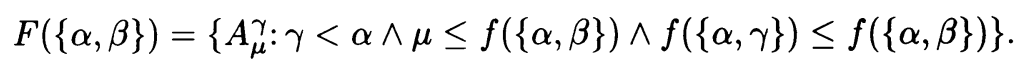

Then $|F(\{\alpha, \beta\})| \leq \lambda$, because of $(9)$.

Let $D \subset \lambda^{++} \backslash \lambda, \operatorname{tp} D=\lambda^{+}$and $A \in[D]^{\lambda}$. Choose a $\beta \in D$ with $A \subset \beta$. Then $A=A_{\nu}^{\beta}$ for some $\nu<\lambda^{+}$. In case there is an $\alpha \in D \backslash \beta$ with $f(\{\alpha, \beta\}) \geq \nu$, we have $A \in F(\{\alpha, \beta\})$. Hence we may assume that $f(\{\alpha, \beta\})<\nu$ for all $\alpha \in D \backslash \beta$. By (8), there are $\alpha^{\prime}<\alpha, \alpha^{\prime}, \alpha \in D \backslash \beta$ such that $f\left(\left\{\alpha, \alpha^{\prime}\right\}\right) \geq \nu$. But, then $f(\{\alpha, \beta\}) \leq f\left(\left\{\alpha, \alpha^{\prime}\right\}\right)$, hence $A \in F\left(\left\{\alpha, \alpha^{\prime}\right\}\right)$.

Theorem 1. Assume $2^{\lambda}=\lambda^{+}$for some $\lambda \geq \omega$. Then Black wins $R\left(\lambda^{+}, 2, \lambda^{+}\right)$.

ProOF. By Lemmas 0 and 1.

THEOREM 2. Assume $2^{\lambda}=\lambda^{+}$for some $\lambda \geq \omega$ and $\lambda^{++} \nrightarrow\left[\lambda^{+}\right]_{\lambda^{+}, \lambda}^{2}$ holds. Then Black wins $R\left(\lambda^{++}, 3, \lambda^{+}\right)$.

ProOF. By Lemmas 1 and 2.

For a cardinal $\lambda \geq \omega$ let $\lambda^{+\alpha}$ be the $\alpha$ th successor of $\lambda$. $\left(\lambda^{+0}=\lambda, \lambda^{+1}=\lambda^{+}\right)$. 
LEMMA 3. Assume $\lambda \geq \omega, 1 \leq r<\omega$. Then

$$
\operatorname{Free}_{\lambda}^{*}\left(\lambda^{+r}, r, \lambda^{+}, \lambda\right) \Leftrightarrow \operatorname{Free}_{\lambda}^{*}\left(\lambda^{+r},<\omega, \lambda^{+}, \lambda\right) \text {. }
$$

This can be shown with a standard argument used e.g. for proving

$$
\aleph_{2} \rightarrow\left[\aleph_{1}\right]_{\aleph_{1}, \aleph_{0}}^{2} \Leftrightarrow \aleph_{2} \rightarrow\left[\aleph_{1}\right]_{\aleph_{1}, \aleph_{0}}^{<\omega} .
$$

We omit the proof. Lemma 3 is just stated to explain our interest in the following

Problem 5. Is $\forall \kappa \geq \omega \neg \operatorname{Free}_{\omega}^{*}\left(\kappa,<\omega, \omega_{1}, \omega\right)$ consistent with G.C.H.? Does it follow from $V=L$ ?

We conjecture that $\exists \kappa \operatorname{Free}_{\omega}^{*}\left(\kappa,<\omega, \omega_{1}, \omega\right)$ implies that $0 \#$ exists. Note that a yes answer to Problem 5 would imply, by Lemmas 1 and 3 , that the following is consistent with ZFC and G.C.H.:

$$
\forall 1 \leq r<\omega \text { Black wins } R\left(\aleph_{r}, r+1, \aleph_{1}\right) .
$$

We now turn our attention to the case when $\kappa$ is a singular strong limit cardinal.

THEOREM 3. Assume $\kappa$ is a singular strong limit cardinal. Then White wins the game $R(\kappa, 2, \kappa)$.

PROOF. Let $\tau=\operatorname{cf}(\kappa)$. To prepare his strategy White first chooses a strictly increasing sequence of cardinals $\left(\kappa_{\nu}: \nu<\tau\right)$ such that $\tau<\kappa_{\nu}<\kappa, \kappa_{\nu}$ is regular for $\nu<\tau$, and the sequence $\left(2^{\kappa_{\nu}}: \nu<\tau\right)$ is strictly increasing as well. For $\nu<\tau$ write $\lambda_{\nu}=\left(2^{\kappa_{\nu}}\right)^{+}$. Now White fixes pairwise disjoint sets $\left\{A_{\nu}: \nu<\tau\right\}$ such that $\bigcup\left\{A_{\nu}: \nu<\tau\right\}=\kappa$ and $\left|A_{\nu}\right|=\lambda_{\nu}$ for $\nu<\tau$. White's strategy will come in $\tau$ stages. Assume $\nu<\tau$ and that we have already defined what he has to do in stages $S_{\mu}$ for $\mu<\nu$, in such a way that at each of these stages his strategy requires him to make $\lambda_{\mu}$ moves. Assume further that for each $\mu<\nu$ a subset $B_{\mu} \subset A_{\mu}$ of type $\kappa_{\mu}$ has already been fixed.

Now at the stage $S_{\nu}$ White will play a strategy (with some modifications) described in [2] to obtain a large subset $B_{\nu}$ of $A_{\nu}$ with $\left[B_{\nu}\right]^{2} \subset W$. This strategy comes again in $\lambda_{\nu}$ stages $S_{\nu, \alpha}$ for $\alpha<\lambda_{\nu}$, each stage requiring White to make $2\left(\left(\sum_{\mu<\nu} \kappa_{\mu}\right)+|\alpha|\right)<\lambda_{\nu}$ moves. Assume that $\alpha<\lambda_{\nu}$ and the strategy has already been defined for the stages $S_{\nu, \beta}, \beta<\alpha$, and that different elements $x_{\beta}, y_{\beta} \in A_{\nu} ; \beta<\alpha$ have already been fixed. We imagine that a game is being played according to the rules of $R(\kappa, 2, \cdot)$ and we will denote by $W(Z)=\{\{x, y\}$ : White chose $\{x, y\} \wedge x \in Z\}$. (This is certainly an abuse of notation since $W$ is not defined until the game is finished, but at each stage we know that some ordinals must be in $W$ while some are forced to be left out.) Now at the stage $S_{\nu, \alpha}$ White first fixes an element $x_{\alpha}$ of $A_{\nu}$ such that $x_{\alpha}>x_{\beta}, y_{\beta}$ for $\beta<\alpha$, and no pair $\left\{x_{\alpha}, \mu\right\}$ has been previously picked either by White or by Black. This can be done since $\lambda_{\nu}$ is greater than the number of moves made so far. Then making $\sum_{\mu<\nu} \kappa_{\mu}$ moves he makes sure that $W\left(\left\{x_{\alpha}\right\}\right) \cap B_{\mu}$ contains a closed cofinal subset of $B_{\mu}$ for each $\mu<\nu$. This is possible since he can always choose $\left\{y, x_{\alpha}\right\}$ for the smallest element $y$ of some $B_{\mu}$ in question which was previously not chosen. At this point it is used heavily that at limit stages it is always White's turn to move. Note that "closed cofinal" is meant in the topology of $B_{\mu}$ defined by its natural well-ordering. After having finished with $x_{\alpha}$, White fixes a new element $y_{\alpha}>x_{\beta}, \beta \leq \alpha ; y_{\alpha}>y_{\beta}, \beta<\alpha$, and repeats the above procedure for $y_{\alpha}$ in $\sum_{\mu<\nu} \kappa_{\mu}$ moves. After this White turns his attention to the set $A_{\nu}$. He now plays at most $2|\alpha|$ moves the following way. If at a 
nonlimit ordinal $\rho+1$, Black at his $\rho$ th move picked a set $\left\{x_{\beta}, x_{\alpha}\right\}$ or $\left\{y_{\beta}, y_{\alpha}\right\}$ with some $\beta<\alpha$, then White picks $\left\{y_{\beta}, y_{\alpha}\right\}$ or $\left\{x_{\beta}, x_{\alpha}\right\}$, respectively, if that is possible, and in any other case he picks an $\left\{x_{\beta}, x_{\alpha}\right\}$ or a $\left\{y_{\beta}, y_{\alpha}\right\}$ with the smallest possible $\beta$. The stage $S_{\nu, \alpha}$ is finished if all the $\left\{x_{\beta}, x_{\alpha}\right\},\left\{y_{\beta}, y_{\alpha}\right\}, \beta<\alpha$, have been chosen by one of the players. We now still have to tell which set $B_{\nu} \subset A_{\nu}$ White should fix. The definition of this set will describe White's strategy but in the meantime it will almost accomplish the proof of the fact that the strategy indeed works. After having finished the stage $S_{\nu}$ White defines a set mapping $F$ on $\lambda_{\nu}$ as follows. For $\alpha<\lambda_{\nu}, F(\alpha)=\left\{\beta<\alpha:\left\{x_{\beta}, x_{\alpha}\right\} \wedge\left\{y_{\beta}, y_{\alpha}\right\}\right.$ were both picked by Black $\}$. By the strategy described above $|F(\alpha)| \leq 2\left(\sum_{\mu<\nu} \kappa_{\mu}\right) \leq \kappa_{\nu}$. Now $\kappa_{\nu}^{+} \leq 2^{\kappa_{\nu}}<\lambda_{\nu}$, hence there is a subset $C \in\left[\lambda_{\nu}\right]^{\lambda_{\nu}}$ Free for $F$.

For each $\alpha \in C$ let $D_{\alpha}=W\left(\left\{x_{\alpha}\right\}\right) \cap W\left(\left\{y_{\alpha}\right\}\right)$. Note that $D_{\alpha} \cap B_{\mu}$ contains a closed unbounded subset of $B_{\mu}$ for each $\mu<\nu$. Let $\hat{B}_{\nu}=\bigcup\left\{B_{\mu}: \mu<\nu\right\}$. Since $\left|\hat{B}_{\nu}\right| \leq \kappa_{\nu}$ there is a $C^{\prime} \in[C]^{\lambda_{\nu}}$ and a subset $D^{\nu} \subset B_{\nu}$ such that $D^{\nu}=\hat{B}_{\nu} \cap D_{\alpha}$ for $\alpha \in C^{\prime}$. Now White defines a 2-partition of length 2 of $C^{\prime}$ as follows. For $\beta<\alpha, \alpha, \beta \in C^{\prime}$,

$$
f(\{\alpha, \beta\})=0 \quad \text { iff }\left\{x_{\alpha}, x_{\beta}\right\} \in W .
$$

Note that, by the definition of $C^{\prime}, f(\{\alpha, \beta\})=1$ implies $\left\{y_{\alpha}, y_{\beta}\right\} \in W$. Now, by the Erdös-Rado theorem $\left(2^{\kappa_{\nu}}\right)^{+} \rightarrow\left(\kappa_{\mu}^{+}\right)_{\kappa_{\nu}}^{2}$, there is a subset $C^{\prime \prime} \subset C^{\prime},\left|C^{\prime \prime}\right|=\kappa_{\nu}^{+}$ homogeneous for $f$. To make the computation simpler White only chooses a $C^{\prime \prime}$ of type $\kappa_{\nu}$ of this kind. Now one of the sets $\left\{x_{\alpha}: \alpha \in C^{\prime \prime}\right\},\left\{y_{\alpha}: \alpha \in C^{\prime \prime}\right\}$ is such that all pairs of the set are in $W$. White will call this set $B_{\nu}$. Note that $\operatorname{tp} B_{\nu}=\kappa_{\nu}$.

Considering that $\tau<\kappa_{0}$, for each $\mu<\tau, B_{\mu}^{\prime}=B_{\mu} \cap \bigcap\left\{D^{\nu}: \mu<\nu<\tau\right\}$ contains a closed unbounded subset of $B_{\mu}$, hence $\left|B_{\mu}^{\prime}\right|=\kappa_{\mu}$.

But then for $B=\bigcup\left\{B_{\mu}^{\prime}: \mu<\tau\right\},|B|=\kappa$ and $[B]^{2} \subset W$.

2. The game $R(\kappa,<\omega, \beta)$. Sequoias. First we deal with the winning strategies of Black.

LEMMA 4. Assume $\neg$ Free $_{1}(\kappa,<\omega, \beta)$ holds for a limit ordinal $\beta \leq \kappa$. Then there is a family $F=\left\{\left\{X_{\alpha}, Y_{\alpha}\right\}: \alpha<\kappa\right\}$ of pairs of finite subsets of $\kappa$, such that $X_{\alpha} \stackrel{\ulcorner}{\neq} Y_{\alpha}$ for $\alpha<\kappa,\left\{X_{\alpha}, Y_{\alpha}\right\} \cap\left\{X_{\gamma}, Y_{\gamma}\right\}=\emptyset$ for $\alpha \neq \gamma<\kappa$ and $\forall A \subset \kappa(\operatorname{tp} A=$ $\left.\beta \Rightarrow \exists \alpha<\kappa, Y_{\alpha} \subset A\right)$.

PROOF. Let $f:[\kappa]^{<\omega} \rightarrow \kappa$ establish the assumption, i.e. $\forall X \in[\kappa]^{<\omega} f(X) \notin$ $X \wedge \forall A \subset \kappa\left(\operatorname{tp} A=\beta \Rightarrow \exists X \in[A]^{<\omega} f(X) \in A\right)$.

For $X \in[\kappa]^{<\omega}$ let $n(X)=|f(X) \cap X|$. Note that $|X|=n$ implies that $n(X) \leq$ $n$. Let $\varphi$ be a one-to-one mapping of $\omega^{2}$ into the set of even numbers and such that $\varphi(n, m) \geq n$. We define a new mapping $g:[\kappa]^{<\omega} \rightarrow \kappa$ which also establishes $\neg$ Free $_{1}(\kappa,<\omega, \beta)$ as follows.

Let $Y \in[\kappa]^{l}$. Let $(n, m)=\varphi^{-1}(\{l\})$ if $l \in R(\varphi)$. Let $X$ be the set of first $n$ elements of $Y$. If $f(X) \in \bigcap(Y \backslash X)$ and $n(X)=m$ let $g(Y)=f(X)$. Let $g(Y)=0$ otherwise.

Let $F=\left\{\{X, Y\}: X \in[\kappa]^{<\omega} \wedge Y=X \cup\{g(X)\} \wedge g(X) \neq 0\right\}$.

Clearly $X \varsubsetneqq Y$ holds for all $\{X, Y\} \in F$. Assume $D \subset \kappa$, tp $D=\beta$. We may assume $0 \notin D$. There is a $Z \subset D, Z \in[D]^{<\omega}$ with $f(Z) \in D$. Let $l=\varphi(|Z|, n(Z))$. Choose an endextension $X$ of $Z$ with $|X|=l$ and $X \subset D$ such that $f(Z) \in \bigcap X \backslash Z$. 
This is possible since $\beta$ is a limit ordinal. Let $Y=X \cup\{g(X)\}=X \cup\{f(Z)\} \subset D$. Obviously $\{X, Y\} \in F$. Finally let $\{X, Y\},\left\{X^{\prime}, Y^{\prime}\right\}, X \subset Y, X^{\prime} \subset Y^{\prime}$, be two elements of $F$. Assume

$$
\{X, Y\} \cap\left\{X^{\prime}, Y^{\prime}\right\} \neq \emptyset .
$$

Then either $X=X^{\prime}$ or $Y=Y^{\prime}$ since the $X^{\prime}$ 's have an even number of elements while the $Y$ 's have an odd number of elements. $X=X^{\prime}$ implies $Y=Y^{\prime}$. On the other hand assume $Y=Y^{\prime},|Y|=l+1$. Since $0 \notin Y, l \in R(\varphi)$ and for $\varphi^{-1}(\{l\})=(n, m), X \cup\{g(X)\}=X^{\prime} \cup\left\{g\left(X^{\prime}\right)\right\}=Y$, and for $Z, Z^{\prime}$, the set of first $n$ elements of $X$ and $X^{\prime}$, respectively, we have $n(Z)=n\left(Z^{\prime}\right)=m$ and $n(X), n\left(X^{\prime}\right) \leq n$. This implies that both $f(Z)$ and $f\left(Z^{\prime}\right)$ are the $j$ th elements of $Y$ for the same $j \leq n+1$. It follows that $X=X^{\prime}$.

ThEOREM 4. Assume $\neg \operatorname{Free}_{1}(\kappa,<\omega, \beta)$ for a limit ordinal $\beta$. Then Black wins the game $R(\kappa,<\omega, \beta)$.

Proof. Consider a system $F=\left\{\left\{X_{\alpha}, Y_{\alpha}\right\}: \alpha<\kappa\right\}$ satisfying the requirements of Lemma 4. Black's strategy is the following. Whenever White chooses an element of $\left\{X_{\alpha}, Y_{\alpha}\right\}$ for some $\alpha<\kappa$ Black chooses the other element of it.

We now start to give the preliminaries needed to obtain winning strategies for White.

DEFInition. Let $\kappa$ be any cardinal. For $X, Y \subset \kappa$ we write $X \prec Y$ iff $\forall \alpha \in$ $X, \forall \beta \in Y, \alpha<\beta$.

(i) A subset $S \subset[\kappa]^{<\omega}$ is said to be a sequoia (on $\kappa$ ) if it has the following two properties:

(a) $S$ is hereditary, i.e. $a \subset b \in S \Rightarrow a \in S$.

(b) $S$ possesses the following exchange property: For $a, b \in[\kappa]^{<\omega}, b \prec\{\beta\} \prec$ $\{\alpha\} \prec a, a \cup\{\alpha\} \cup\{\beta\} \in S$ imply that

$$
a \cup\{\alpha\} \cup b \in S \Leftrightarrow a \cup\{\beta\} \cup b \in S .
$$

REMARKS. $S_{0}=\emptyset, S_{1}=[\kappa]^{<\omega}$ are sequoias on $\kappa$. Let $\left\langle\kappa, \prec^{\prime}\right\rangle$ be a tree on $\kappa$, such that $\prec^{\prime} \subset \prec$. Let $S_{2}=\left\{a \in[\kappa]^{<\omega}: a\right.$ is fully ordered by $\left.\prec^{\prime}\right\}$. Then $S_{2}$ is a sequoia on $\kappa$. On the other hand let $S$ be a sequoia. Put $\alpha \prec^{\prime} \beta \Leftrightarrow\{\alpha, \beta\} \in S \wedge \alpha<\beta$. Then $\left\langle\kappa, \prec^{\prime}\right\rangle$ is a tree. Let $B$ be a branch of this tree. For $\alpha<\beta, \alpha, \beta \in B$ define $\alpha \prec_{B}^{2} \quad \beta \Leftrightarrow \exists \gamma, \beta<\gamma \in B,\{\alpha, \beta, \gamma\} \in S$. Then, by the exchange property, $\alpha \prec_{B}^{2} \beta \Leftrightarrow \forall \gamma, \beta<\gamma<B,\{\alpha, \beta, \gamma\} \in S$, and again by the exchange property $\left\langle B,{ }_{B}^{2}\right\rangle$ is a tree. Obviously this procedure can be continued, and a sequoia is a giant collection of trees amalgamated by the exchange property.

(ii) For a sequoia $S$ and for $a \in S$ let $S_{a}=\{b \in S: b \prec a \wedge a \cup b \in S\}$.

(iii) For a sequoia $S$ and $a \in S$ let $a^{-}=a \backslash\{\min a\}$ if $a \neq 0.0^{-}=0$. We define

$$
\operatorname{Twin}(S, a)=\left\{\beta:\{\beta\} \prec a \wedge a^{-} \cup\{\beta\} \in S \wedge S_{a}=S_{a^{-} \cup\{\beta\}}\right\} .
$$

Note that $\operatorname{Twin}(S, \emptyset)=\emptyset$, and $\operatorname{Twin}(S, a) \prec a$ for all $a \in S$.

(iv) We say that $a \in S$ is normal in $S$ if $|\operatorname{Twin}(S, a)| \leq 1$ and $\operatorname{Twin}(S, a)=\emptyset$ provided $\bigcup S_{a} \neq \emptyset$ and $\bigcup S_{a}$ does not have a maximal element and $S$ is a normal sequoia if all elements $a$ of it are normal in $S$.

Note that in the example $S_{2}$, the set $\{\alpha\}$ is normal in $S_{2}$ iff there is at most one other $\beta<\alpha$ with $\hat{\alpha}=\hat{\beta}$, and none if the height of $\alpha$ is a limit ordinal. Hence $S_{2}$ is a normal sequoia iff $\left\langle\kappa,<^{\prime}\right\rangle$ is a normal binary tree. It is also easy to see that 
a normal sequoia is an amalgamation of normal binary trees as indicated in the remark made after definition (i).

Finally for the construction we need two more definitions:

(v) We will denote by $<^{*}$ the natural well-ordering (of type $\kappa$ ) of $[\kappa]^{<\omega}$.

For $a \in[\kappa]^{n}$ let $a=\left(a_{0}, \ldots, a_{n-1}\right), a_{0}>\cdots>a_{n-1}, a<^{*} b \Leftrightarrow a$ is an endsection of $b \vee b_{j}>a_{j}$ for the minimal $j$ with $b_{j} \neq a_{j}$.

Finally we say that

(vi) $S$ is an almost normal sequoia provided for all $a \in S$, $a$ is either normal in $S$ or $\forall b \in S, a<^{*} b$ implies that $a$ is an endsection of $b$, i.e. $\exists c \prec a$ with $c \cup a=b$.

We now need a sequence of lemmas about the properties of sequoias. In these lemmas $S$ always denotes a sequoia.

S. LEMMA 1. For $a \in S, S_{a}$ is a sequoia.

Proof. (a) Assume $b \subset c \in S_{a}$. Then $c \cup a \in S, c \prec a, b \cup a \in S$, and so $b \in S_{a}$.

(b) Assume $b \cup\{\alpha, \beta\} \in S_{a}, c \prec\{\beta\} \prec\{\alpha\} \prec b$. Then $a \cup b \cup\{\alpha, \beta\} \in S$ and the exchange property for $S$ implies the exchange property for $S_{a}$.

S. LEMMA 2. Assume $\emptyset \neq b \in S_{a}$. Then $b$ is normal in $S_{a}$ iff $b \cup a$ is normal in $S$.

PROOF. $b \prec a$ by the assumption. Note that $\left(S_{a}\right)_{b}=S_{a \cup b}$ and thus $\bigcup\left(S_{a}\right)_{b}$ has a maximal element if $\bigcup S_{a \cup b}$ has one. Finally

$$
\begin{aligned}
\operatorname{Twin}\left(S_{a}, b\right) & =\left\{\beta:\{\beta\} \prec b \wedge\left(S_{a}\right)_{b-\cup\{\beta\}}=\left(S_{a}\right)_{b}\right\} \\
& =\left\{\beta:\{\beta\} \prec b \wedge S_{(a \cup b)-\cup\{\beta\}}=s_{a \cup b}\right\}=\operatorname{Twin}(S, a \cup b) .
\end{aligned}
$$

S. LEMMA 3. Assume $S$ is almost normal. Then it has at most one element a which is not normal in $S$.

Proof. Assume indirectly that $a, b \in S$ and $a, b$ are not normal in $S$. W.l.o.g. we may assume $a<^{*} b$. Then $S$ being almost normal, $a$ is an endsection of $b$, i.e. there is a $c \prec a$ with $b=a \cup c$ and $c \neq \emptyset$. Now, by S. Lemma 2, $c$ is not normal in $S_{a}$. Using again that $a$ is not normal in $S$, we can choose a $\{\alpha\} \prec a$ with $\alpha \in \operatorname{Twin}(S, a)$. Then $S_{a^{-} \cup\{\alpha\}}=S_{a}$. Hence $c$ is not normal in $S_{a^{-} \cup\{\alpha\}}$ and then, by S. Lemma 2, $a^{-} \cup\{\alpha\} \cup c$ is not normal in $S$. Now $a^{-} \cup\{\alpha\} \cup c<^{*} a$ and $a^{-} \cup\{\alpha\} \cup c \not \subset a$. This contradicts the fact that $S$ is almost normal.

S. LEMMA 4. Assume $S$ is almost normal and $a \in S$ is not normal in $S$. Then $\max a=\max \bigcup S$. As a corollary of this if $\bigcup S$ has no maximal element, then $S$ is normal.

Proof. Assume $\max a<\beta$ for some $\beta \in \bigcup S$. Then $a<^{*}\{\beta\}$ and $a \not \subset\{\beta\}$, hence $S$ is not almost normal.

S. LEMMA 5. Let $\eta$. be a limit ordinal. Let $\left\{S_{\xi}: \xi<\eta\right\}$ be a sequence of almost normal sequoias satisfying the following condition. For all $\xi<\eta$ there is an $a_{\xi} \in S_{\xi+1}$ such that

$$
S_{\xi}=\left\{b \in S_{\xi+1}: b<^{*} a_{\xi}\right\} .
$$

Then $S=\bigcup\left\{S_{\xi}: \xi<\eta\right\}$ is an almost normal sequoia.

Proof. (a) $S$ is hereditary: Let $a \subset b \in S$. Then $b \in S_{\xi}$ for some $\xi<\eta, a \in S_{\xi}$ and $a \in S$. 
(b) $S$ has the exchange property: Assume $\{\beta\} \prec\{\alpha\} \prec a, a \cup\{\alpha\} \cup\{\beta\} \in S$. Then for some $\xi<\eta, a \cup\{\alpha\} \cup\{\beta\} \in S_{\xi}$, hence $a \cup\{\alpha\} \cup\{\beta\}<^{*} a_{\xi}$. Now, for any $b \prec\{\beta\}, a \cup\{\alpha\} \cup b$,

$$
a \cup\{\beta\} \cup b<^{*} a \cup\{\alpha\} \cup\{\beta\}<^{*} a_{\xi}
$$

holds. It now follows that

$$
\begin{aligned}
a \cup\{\alpha\} \cup b \in S & \Leftrightarrow a \cup\{\alpha\} \cup b \in S_{\xi} \\
& \Leftrightarrow a \cup\{\beta\} \cup b \in S_{\xi} \Leftrightarrow a \cup\{\beta\} \cup b \in S .
\end{aligned}
$$

Finally, we have to see that $S$ is almost normal. Let $\emptyset \neq a \in S$. We distinguish two cases.

(i) $\exists \xi<\eta\left(a<^{*} a_{\xi} \wedge a\right.$ is not an endsection of $\left.a_{\xi}\right)$.

(ii) $\exists \xi_{0}<\eta, \forall \xi_{0}<\xi<\eta\left(a\right.$ is an endsection of $a_{\xi}$ ).

In case (i), $S_{\xi+1}$ being almost normal, $a$ is normal in $S_{\xi+1}$. Again, by (i) $\forall b \prec a\left(a \cup b<^{*} a_{\xi}\right)$. Hence $S_{a}=\left\{b \prec a: a \cup b \in S \wedge a \cup b<^{*} a_{\xi}\right\}=\left(S_{\xi}\right)_{a} \subset$ $\left(S_{\xi+1}\right)_{a} \subset S_{a}$, and as a corollary of this $S_{a}=\left(S_{\xi+1}\right)_{a}$. Similarly, for each $\beta<$ $\min a, S_{a^{-} \cup\{\beta\}}=\left(S_{\xi+1}\right)_{a^{-} \cup\{\beta\}}$. (To see this we have to use the fact that if $a<^{*} a_{\xi}$ and $a$ is not an endsection of $a_{\xi}$ then the same holds for $a^{-} \cup\{\beta\}$.) We now have that $\operatorname{Twin}(S, a)=\operatorname{Twin}\left(S_{\xi+1}, a\right)$ and so the normality of $a$ in $S_{\xi+1}$ implies the normality of $a$ in $S$.

In case (ii) let $b \in S, a<^{*} b$ be arbitrary. For some $\xi, \xi_{0}<\xi<\eta, b<^{*} a_{\xi}$. $a$ is an endsection of $a_{\xi}$, by (ii), hence the inequalities $a<^{*} b<^{*} a_{\xi}$ imply that $a$ is an endsection of $b$ as well.

S. LEMMA 6. Assume $S$ is almost normal, $a \in S$ is not normal in $S, \beta \in$ Twin $(S, a)$. Then the following statements are true:

(i) $\forall b \in S\left(b<^{*} a \cup\{\beta\}\right)$.

(ii) $S \cup\{a \cup\{\beta\}\}$ is an almost normal sequoia.

ProOF. (i) Let $b \in S$. Since $a<^{*} a \cup\{\beta\}$ we may assume $a<^{*} b$. Because $a$ is not normal in $S$, then there exists a $c \neq \emptyset$ with $a \cup c=b, c \prec a$. This implies that $c \in S_{a}=S_{a^{-} \cup\{\beta\}}$. Hence $c \prec\{\beta\}$ and as a consequence of it $b=a \cup c<^{*} a \cup\{\beta\}$.

(ii) (a) $S \cup\{a \cup\{\beta\}\}$ is hereditary: To prove this it is sufficient to see that for all $b \subset a \cup\{\beta\}$ with $|a \cup\{\beta\} \backslash b|=1, b \in S$ holds. Assume $b \notin S$ for some of these $b$. For such $b, a \cup\{\beta\}=b \cup\{\alpha\}$ for some ordinal $\alpha$, and let $\alpha$ be the minimal ordinal for which $b \in S$ fails. Then $\alpha \neq \beta$ since $a \in S$ and $\alpha \neq \min a$ since $a^{-} \cup\{\beta\} \in S$. Then $a \cup\{\beta\}$ can be written in the form $a \cup\{\beta\}=a^{\prime} \cup\{\alpha\} \cup\left\{\alpha^{\prime}\right\} \cup a^{\prime \prime} \cup\{\beta\}$ where $\{\beta\} \prec a^{\prime \prime} \prec\left\{\alpha^{\prime}\right\} \prec\{\alpha\} \prec a^{\prime}$. Then $a^{\prime} \cup\{\alpha\} \cup\left\{\alpha^{\prime}\right\} \subset a \in S$, and $a^{\prime} \cup\{\alpha\} \cup a^{\prime \prime} \cup\{\beta\} \in S$ because of the minimality of $\alpha$. Then $b \in S$ since $S$ possesses the exchange property.

(b) $S \cup\{a \cup\{\beta\}\}$ has the exchange property.

Put $S^{\prime}=S \cup\{a \cup\{\beta\}\}$. Using part (i) of S. Lemma 6 it is easy to see that $S^{\prime}$ has the exchange property provided

$$
\forall b \prec\{\beta\} a^{-} \cup b \cup\{\beta\} \in S \Leftrightarrow a \cup b \in S .
$$

This is equivalent to $S_{a-\cup\{\beta\}}=S_{a} \cap[\beta]^{<\omega}$ which is true because $\beta \in \operatorname{Twin}(S, a)$.

(c) We have to prove that $S^{\prime}$ is almost normal.

Let $b \in S^{\prime}$. If $a \cup\{\beta\}$ is not of the form $a \cup\{\beta\}=b \cup c, c \prec b$ then $S_{b}^{\prime}=$ $S_{b}, \operatorname{Twin}\left(S^{\prime}, b\right)=\operatorname{Twin}(S, b)$, hence $b$ remains normal in $S^{\prime}$. Assume now $a \cup\{\beta\}=$ 
$b \cup c, c \prec b$. We claim that $\forall d \in S^{\prime}\left(b<^{*} d \Rightarrow b\right.$ is an endsection of $\left.d\right)$. This indeed follows from the fact that $a$ is the nonnormal element of $S$.

Note that the proof also shows that the nonnormal element of $S^{\prime}$ (if any) must be an endsection of $a \cup\{\beta\}$.

We are now in a position to prove our main theorem concerning Ramsey games and sequoias.

THEOREM 5. Assume $\kappa \geq \omega$ is a cardinal. Then in the game $R(\kappa,<\omega, \cdot)$ White has a strategy to achieve that its winning set $W$ contains a normal sequoia $S$ with $|\bigcup S|=\kappa$.

ProOF. White's first move is to choose the empty set. We define White's strategy recursively. We will denote White's and Black's $\xi^{\prime}$ th move by $a_{\xi}$ and $b_{\xi}$, respectively. We want to define White's strategy so that the following conditions should hold in every game played according to this strategy for every $\xi<\kappa$ :

(i) $a_{\zeta}<{ }^{*} a_{\xi}$ for $\zeta<\xi$.

(ii) $S_{\xi}=\left\{a_{\zeta}: \zeta<\xi\right\}$ is an almost normal sequoia.

(iii) In case $S_{\xi}$ is normal, $a_{\xi}=\left\{\beta_{\xi}\right\}$ for $\beta_{\xi}=\min \left(\kappa \backslash\left(\bigcup\left\{a_{\zeta} \cup b_{\zeta}: \varsigma<\xi\right\}\right)\right)$.

(iv) In case $S_{\xi}$ is almost normal and $a_{f(\xi)}$ is the nonnormal element of $S_{\xi}(f(\xi)<$ $\xi)$, then $a_{\xi}=a_{f(\xi)} \cup\left\{\beta_{\xi}\right\}$ for some $\beta_{\xi} \in \operatorname{Twin}\left(S_{\xi}, a_{f(\xi)}\right)$.

Let $T_{\xi}=\operatorname{Twin}\left(S_{\xi}, a_{f(\xi)}\right)$ in case $S_{\xi}$ is not normal.

(v) If $S_{\xi+1}$ is not normal, then $\left|T_{\xi+1}\right| \geq 2$. Furthermore, suppose $b_{\xi}$ is of the form $b_{\xi}=b \cup\{\alpha\}$, where $b \neq \emptyset,\{\alpha\} \prec b$ and $a_{f(\xi+1)}=b \cup c$ for some $c \prec\{\alpha\}$ and that $\min T_{\xi+1}<\alpha$. Then we call $b_{\xi}$ a threat and in this case we denote the set $b^{-} \cup\{\alpha\} \cup c \cup\{\beta\}$ by $d(\beta)$ for $\beta \in T_{\xi+1}$ and we require that either

(a) $d\left(\beta_{\xi+1}\right) \notin S_{\xi+1}$ or

(b) $\beta_{\xi+1}>\min T_{\xi+1}$ and for all $\beta \leq \beta_{\xi+1}$ with $\beta \in T_{\xi+1}, d(\beta) \in S_{\xi+1}$.

Assume now that $0<\eta<\kappa$ and the strategy has already been defined so that any game $\left(a_{\xi}, b_{\xi}: \xi<\eta\right)$ played according to White's strategy satisfies (i)-(iv) for $\xi<\eta$ and $(\mathrm{v})$ for $\xi+1<\eta$.

Since any move $b_{\xi}$ of Black prevents White from choosing any superset of $b_{\xi}$ we may assume that Black's moves are minimal, i.e. any proper subset $a$ of $b_{\xi}$ is equal to some $a_{\varsigma}$ with $\zeta \leq \xi$.

Now $S_{\eta}=\left\{a_{\xi}: \xi<\eta\right\}$ is defined, and, by S. Lemmas 5 and $6, S_{\eta}$ is an almost normal sequoia. In case $S_{\eta}$ is normal (iii) can be trivially satisfied. (If $S$ is a normal sequoia and $\alpha \prec\{\beta\}$ for all $a \in S$, then $S \cup\{\{\beta\}\}$ is an almost normal sequoia.) We assume now that $S_{\eta}$ is not normal and $a_{f(\eta)}$ is the nonnormal element of it. By S. Lemma 6 to finish the recursion we only have to show that $\beta_{\eta}$ can be chosen to satisfy (iv) and (v).

To see this we first prove the following

Claim. For $\alpha \in T_{\eta}$ and $\xi+1<\eta, a_{f(\eta)} \cup\{\alpha\} \neq b_{\xi}$.

Assume indirectly that $a_{f(\eta)} \cup\{\alpha\}=b_{\xi}$. By the minimality of the $b_{\xi}$ 's, $a_{f(\eta)} \in$ $S_{\xi+1}$, i.e. $f(\eta) \leq \xi$. Then $a_{f(\eta)}<{ }^{*} a_{\xi+1} \in S_{\eta}$, because of (i), hence by the choice of $a_{f(\eta)}$ and the almost normality of $S_{\eta}, a_{f(\eta)}$ is an endsection of $a_{\xi+1}=$ $a_{f(\xi+1)} \cup\left\{\beta_{\xi+1}\right\}$. We intend to show now that $b_{\xi}$ was a threat. Since $f(\eta) \leq$ $\xi, a_{f(\eta)} \neq a_{\xi+1}$ and so $a_{f(\eta)}$ is an endsection of $a_{f(\xi+1)}$ as well. Then denoting $a_{f(\eta)}$ by $b, a_{f(\xi+1)}=b \cup c$ for some $c \prec b$. 
By S. Lemma 6, $a_{f(\xi+1)}<^{*} a_{f(\eta)} \cup\{\alpha\}=b \cup\{\alpha\}$. This implies that $\alpha>\max c$ for $c \neq \emptyset$. In case $c=\emptyset, a_{f(\xi+1)} \cup\left\{\beta_{\xi+1}\right\}=a_{\xi+1}<^{*} a_{f(\eta)} \cup\{\alpha\}$ implies $\beta_{\xi+1}<\alpha$. Hence $b_{\xi}$ was indeed a threat. By S. Lemma $6, S^{\prime}=S_{\eta} \cup\left\{a_{f(\eta)} \cup\{\alpha\}\right\}$ is a sequoia, and it has the exchange property. It follows from the requirements on a threat and from $\beta_{\xi+1}<\alpha$ that the exchange property yields

$$
d(\beta) \in S^{\prime} \Leftrightarrow a_{f(\xi+1)} \cup\{\beta\} \in S^{\prime} \text { for } \beta \leq \beta_{\xi+1} \text { and } \beta \in T_{\xi+1} .
$$

Now we apply (v) for $\xi+1$. If White answered the threat by making sure $d\left(\beta_{\xi+1}\right) \notin S$ we get a contradiction from (10) since then $a_{f(\xi+1)} \cup\{\beta\} \in S^{\prime}$, and $d\left(\beta_{\xi+1}\right) \in S^{\prime}$. But $d\left(\beta_{\xi+1}\right)<{ }^{*} b \in S_{\xi+1}$ and so $d\left(\beta_{\xi+1}\right) \in S_{\xi+1}$.

If White answered the threat the other way, and $\bar{\beta}=\min T_{\xi+1}$, then the exchange property (10) implies $a_{f(\xi+1)} \cup\{\bar{\beta}\} \in S^{\prime}$. But $a_{f(\xi+1)} \cup\{\bar{\beta}\}<^{*} a_{\xi+1}$ then implies $a_{f(\xi+1)} \cup\{\bar{\beta}\} \in S_{\dot{\xi}+1}$, a contradiction to the choice of $\beta_{\xi+1}$ and to S. Lemma 6 . This contradiction finally proves the claim.

By the choice of $a_{f(\eta)}, T_{\eta} \neq \emptyset$. Hence if $\eta$ is a limit ordinal and $\beta \in T_{\eta}$, by the claim just proved, $a_{\eta}=a_{f(\eta)} \cup\{\beta\}$ is a legal move for White. Choosing $\beta_{\eta}$ to be the minimal $\beta$ of this kind, the $\eta$ 's move of the strategy is defined and satisfies (iv) (and (v) vacuously). Assume now $\eta=\xi+1$. Since $f(\xi+1) \leq \xi, a_{f(\xi+1)} \leq{ }^{*} a_{\xi}$. In case $a_{f(\xi+1)}=a_{\xi}, a_{\xi}$ being the $<^{*}$ maximal element of $S_{\xi+1},\left(S_{\eta}\right)_{a_{f(\eta)}}=$ $\{\emptyset\}, \bigcup\left(S_{\eta}\right)_{a_{f(\eta)}}=\emptyset$; hence $\left|T_{\eta}\right| \geq 2$. In case $a_{f(\eta)}<{ }^{*} a_{\xi}, a_{\xi}=a_{f(\eta)} \cup d$ for some $\emptyset \neq d \prec a_{f(\eta)}$. Again, by the maximality of $a_{\xi}, \max d=\max \bigcup\left(S_{\eta}\right)_{a_{f(\eta)}}$. Hence $\left|T_{\eta}\right| \geq 2$ holds in this case as well.

By the claim, $b_{\varsigma}=a_{f(\eta)} \cup\{\alpha\}, \zeta<\eta, \alpha \in T_{\eta}$ can only hold for $\zeta=\xi$. In case this holds for say $\alpha_{0} \in T_{\eta}$ (we may assume $\left|T_{\eta}\right|=2$ ), White is compelled to choose the other element, say $\alpha_{1}$ of $T_{\eta}$, as his $\beta_{\eta}=\beta_{\xi+1}$.

Now in this case, using the notation of $(\mathrm{v}), a_{f(\xi+1)}=b, c=\emptyset$. Hence in case $\alpha_{0}<\alpha_{1}, b_{\xi}$ is not a threat and the rest of $(\mathrm{v})$ holds vacuously. In case $\alpha_{1}<\alpha_{0}, d\left(\beta_{\xi+1}\right)=d\left(\alpha_{1}\right)=a_{f(\eta)}^{-} \cup\left\{\alpha_{0}\right\} \cup\left\{\alpha_{1}\right\} \notin S_{\eta}$ since otherwise $\alpha_{1} \in$ $\left(S_{\eta}\right)_{a_{f(\eta)}^{-} \cup\left\{\alpha_{0}\right\}}, \alpha_{0} \notin\left(S_{\eta}\right)_{a_{f(\eta)}^{-} \cup\left\{\alpha_{1}\right\}}$, and $\alpha_{0}, \alpha_{1} \in T_{\eta}=\operatorname{Twin}\left(S_{\eta}, a_{f(\eta)}\right)$. Hence in this case the only move of White answers the threat and satisfies $(\mathrm{v})$.

Now assume $\alpha_{0}<\alpha_{1}$ are the two smallest elements of $T_{\eta}, b_{\xi} \neq a_{f(\eta)} \cup\left\{\alpha_{i}\right\}, i<2$ and $b_{\xi}$ is a threat: In case $d\left(\alpha_{i}\right) \notin S_{\eta}$ for some $i<2$, White can choose $a_{\eta}=$ $a_{f(\eta)} \cup\left\{\alpha_{i}\right\}$ answering the threat. In case $d\left(\alpha_{i}\right) \in S_{\eta}$ for $i<2, a_{\eta}=a_{f(\eta)} \cup\left\{\alpha_{1}\right\}$ answers the threat.

This finishes the recursion, the strategy is defined. Assume White plays his strategy and makes $\kappa$-moves. Let $S=\left\{a_{\xi}: \xi<\kappa\right\}$. Then $S \subset W$. By (i), (ii) and $\mathrm{S}$. Lemma $5, S$ is an almost normal sequoia, and $|\bigcup S|=\kappa$. By S. Lemma $4, S$ is normal as well.

Now we prove our last lemma about normal sequoias.

S. LEMMA 7. Assume $\kappa \geq \omega, S \subset[\kappa]^{<\omega}$ and $S$ is a normal sequoia. Let $\beta$ be a limit ordinal, $A \subset \bigcup S, \operatorname{tp} A=\beta$ a set of indiscernibles for $S$ (i.e., for a structure coding $S$ and the well-ordering $<$ of $\kappa)$. Then there is $B \subset \kappa, \operatorname{tp} B=\beta$ such that $[B]^{<\omega} \subset S$.

Proof. Let $A=\left\{\alpha_{\xi}: \xi<\beta\right\}$ be the natural enumeration of $A$. We may assume that $\alpha_{0}$ is the minimal ordinal for which a set $A$ of indiscernibles exists 
with $\min A=\alpha_{0}$. If $[A]^{<\omega} \subset S$ we are home. Assume that $k$ is the minimal integer for which $[A]^{k} \not \subset S$. Then $k>1$ and by indiscernibility $[A]^{k} \cap S=\emptyset$.

We now claim that $S_{a}=S_{b}$ for $a, b \in[A]^{k-1}, \min a=\min b$. This is obvious for $k=2$. For $k>2$ assume first that $a=a^{\prime \prime} \cup\{\alpha\} \cup a^{\prime}, b=a^{\prime \prime} \cup\{\beta\} \cup a^{\prime}, a^{\prime} \prec$ $\{\alpha\},\{\beta\} \prec a^{\prime \prime}, a^{\prime} \neq \emptyset$.

Then $\left|a^{\prime \prime} \cup\{\alpha, \beta\}\right| \leq k-1, a^{\prime \prime} \cup\{\alpha, \beta\} \in S$. Hence for any $c \prec a^{\prime}$, by the exchange property,

$$
c \cup a \in S \Leftrightarrow c \cup b \in S, \quad \text { i.e. } S_{a}=S_{b}
$$

holds in this case. Since for any pair $a, b \in[A]^{k-1}$ with $\min a=\min b, b$ can be obtained from $a$ by repeated applications of the operation described above, $S_{a}=S_{b}$ holds for any such pair.

For $\xi<\beta$, let $S(\xi)=S_{\left\{\alpha_{\xi}, \alpha_{\xi+1}, \ldots, \alpha_{\xi+k-2}\right\}}$.

We now claim that $S(\xi) \neq S(\eta)$ holds for every pair $\xi<\eta<\beta$. Indeed otherwise $S(\xi)=S(\eta)$ would hold for all pairs, and as a consequence of this $S_{a}=S_{b}$ would hold for all $a, b \in[A]^{k-1}$. This is impossible since e.g.

$$
\left.S_{\left\{\alpha_{0}, \alpha_{3}, \ldots, \alpha_{k-1}\right\}}\right\}=S_{\left\{\alpha_{1}, \alpha_{3}, \ldots, \alpha_{k-1}\right\}}=S_{\left\{\alpha_{2}, \alpha_{3}, \ldots, \alpha_{k-1}\right\}}
$$

implies that $\alpha_{0}, \alpha_{1} \in \operatorname{Twin}\left(S,\left\{\alpha_{2}, \ldots, \alpha_{k-1}\right\}\right)$ a contradiction to the normality of $S$.

For $\xi<\eta<\beta$, let $a(\xi, \eta)$ be the $<^{*}$ minimal element of $S(\xi) \circ S(\eta)$. Now either (i) $\forall \xi<\eta<\beta, a(\xi, \eta) \in S(\xi) \backslash S(\eta)$ or (ii) $\forall \xi<\eta<\beta, a(\xi, \eta) \in S(\eta) \backslash S(\xi)$ holds. Both of these conditions imply, that $a(\xi, \xi+1) \neq a(\xi+1, \xi+2)$ for $\xi<\beta$, since e.g. if (i) holds then $a(\xi, \xi+1) \notin S(\xi+1)$ and $a(\xi+1, \xi+2) \in S(\xi+1)$.

This in turn implies that $a(\xi, \xi+1)<^{*} a(\xi+1, \xi+2)$ hence the elements $\{a(\xi, \xi+1): \xi<\beta\}$ are all different. Let $a_{j}$ denote the $j$ th element of $a$.

There is a $j<\omega$ such that the elements $\left\{a_{j}(\xi, \xi+1): \xi<\beta\right\}$ are all different and indeed increasing. If (i) holds then $a_{j}(\xi, \xi+1) \in \bigcup S(\xi) \subset \alpha_{\xi}$, for $\xi<\beta$; hence $\left\{a_{j}(\xi, \xi+1): \xi<\beta\right\}$ is a set of indiscernibles with with a smaller minimal element than $\alpha_{0}$, a contradiction. Thus we may assume that (ii) holds and that $S(\xi) \subset S(\xi+1)$ for $\xi<\beta$.

Let $\beta_{\xi}=\min \bigcup(S(\xi+1) \backslash S(\xi))$ for $\xi<\beta$. In case $\beta_{\xi}<\alpha_{\xi}$ for $\xi<\beta,\left\{\beta_{\xi}: \xi<\beta\right\}$ is a set of indiscernibles with a smaller minimal element than $\alpha_{0} . \beta_{\xi} \neq \alpha_{\xi}$ because of the choice of $k$. It follows that $\alpha_{\xi}<\beta_{\xi}<a_{\xi+1}$ holds for $\xi<\beta$. Now using the fact $\left\{\beta_{\xi}, \alpha_{\xi+1}, \ldots, \alpha_{\xi+k-1}\right\} \in S$ the exchange property for $S$ implies that

$$
\forall b \prec\left\{\beta_{\xi}\right\}\left(b \cup\left\{\beta_{\xi}, \alpha_{\xi_{2}}, \ldots, \alpha_{\xi+k-1}\right\} \in S \Leftrightarrow b \cup\left\{\alpha_{\xi+1}, \alpha_{\xi+2}, \ldots, \alpha_{\xi+k-1}\right\} \in S\right) .
$$

This means that

$$
S_{\left\{\beta_{\xi}, \alpha_{\xi+2}, \ldots, \alpha_{\xi+k-1}\right\}}=\left[\beta_{\xi}\right]^{<\omega} \cap S(\xi+1)
$$

holds for $\xi<\beta$.

Considering that $S(\xi) \subset S(\xi+1)$ and taking into account the definition of $\beta_{\xi}$ it follows that

$$
\left[\beta_{\xi}\right]^{<\omega} \cap S(\xi+1)=S(\xi)=S_{\left\{\alpha_{\xi}, \alpha_{\xi+2}, \ldots, \alpha_{\xi+k-1}\right\}} .
$$

These equalities imply that

$$
\alpha_{\xi} \in \operatorname{Twin}\left(S,\left\{\beta_{\xi}, \alpha_{\xi+2}, \ldots, \alpha_{\xi+k-1}\right\}\right)
$$


and that $\bigcup S_{\left\{\beta_{\xi}, \alpha_{\xi+2}, \ldots, \alpha_{\xi+k-1}\right\}}=\bigcup S(\xi)$ has a maximal element (because of the definition of normality).

Let $g(\xi)=\max \bigcup S(\xi)$ for $\xi<\beta$.

The $g(\xi)$ must be all different, since $\left\{\alpha_{\xi}\right\}<\left\{\beta_{\xi}\right\} \in S(\xi+1)$ holds for $\xi<\beta$.

Then $\{g(\xi): \xi<\beta\}$ is a set of indiscernibles with a smaller maximal element than $\alpha_{0}$. This contradiction concludes the proof of the lemma.

THEOREM 6. Assume $\kappa \rightarrow(\beta)_{2}^{<\omega}$ holds for some limit ordinal $\beta$. Then White wins the game $R(\kappa,<\omega, \beta)$.

Proof. By Theorem 5, White has a strategy which insures that he gets a normal sequoia $S \subset W$ with $|\bigcup S|=\kappa$. By the assumption, there is a set $A \subset$ $\bigcup S, \operatorname{tp} A=\beta$ which is a set of indiscernibles for $A$. Then, by S. Lemma 7 , there is a set $B \subset \kappa, \operatorname{tp} B=\beta$ such that $[B]^{<\omega} \subset S \subset W$.

\section{REFERENCES}

1. J. E. Baumgartner, Results and independence proofs in combinatorial set theory, Doctoral Dissertation, University of California, Berkeley, California, 1970.

2. J. E. Baumgartner, F. Galvin, R. McKenzie and R. Laver, Game theoretic versions of partition relations, Infinite and Finite Sets, Colloquia Math. Soc. János Bolyai, Vol. 10, Keszthely, Hungary, 1973.

3. K. J. Devlin, Some weak versions of large cardinal axioms, Ann. Math. Logic 5 (1973), 291-325.

4. K. J. Devlin and J. B. Paris, More on the free subset problem, Ann. Math. Logic 5 (1973), 327-336.

5. P. Erdös and R. Rado, Combinatorial theorems on classification of subsets of a given set, Proc. London Math. Soc. 3 (1952), 417-439.

6. A. Hajnal, Proof of a conjecture of S. Ruziewriz, Fund. Math. 50 (1961), 123-128.

7. P. Komjath, Preprint.

Department of Mathematics and Statistics, University of Calgary, CalGARY, AlBERTA, CANADA T2N 1N4

Current address (both authors): Department of Logic and Set Theory, Mathematical Institute, Hungarian Academy of Sciences, H-1053 Budapest V, Reáltanoda u. 13-15, Hungary 